\title{
"Study of a Benign Form of Leprosy Localised in the Feet
}

Ramiro Galvez A.

In the Leper Hospital of " La Piedad" in the capital city of Guatemala the author had the opportunity of making a detailed investigation of fourteen cases of a special kind of neural leprosy of a very benign type, whose lesions were very definitely restricted in their localisation in the feet, with no signs of the disease in any other part of the body.

\section{Symptomatology.}

The history is given of the beginnings of the disease as related by the patients, who tell of neuralgia and lightning pains in the feet, cramp of the toes, with a later sensation of heaviness of the feet, formication and numbness of the toes. These sensations were attributed by the patients to all kinds of simple natural causes and they attached no importance to them. Later, however, they noted certain changes in the toe nails, which became dry and brittle, and finally dropped off or disappeared. In others the nails remained, becoming deformed and diminished in size, until they disappeared. Sometimes the interdigital spaces disappeared, the toes becoming fused together into a solid mass. Sometimes the toes assumed the deformity of "hammer toe" during the process of absorption. Sometimes blisters formed with open sores and ulcers. No pain was ever felt, the toes appearing to be without sensation. Other patients told of changes in the skin of the feet, which became dry and hard, thickened and furrowed.

Development of the disease is very slow, but it progresses continuously until a very definite point of limitation is reached. The histories recorded periods of 10 to 20 years. The social status and mentality of the patients did not incline them to take much notice of the very slow process of the mutilation of their feet, which was more or less painless, unless abscesses or infected ulcers caused inconvenience.

Apart from the mechanical inconvenience of the loss of the toes and its effect on their equilibrium and walk, the patients remained quite fit for work, and all their vital organs functioned normally. When there were open sores

*Translated and abstracted by Dr. J. W. Lindsay from a paper read at the 4th Central American Medical Congress, October, 1936. 
and secondary infections the general health would be somewhat affected as in any other (lisease. There was never, however, any general reaction such as is observed in other forms of leprosy from the toxaemia produced by the bacillus of Jansen. Neither was there observed any signs of that conclition of apathy and asthenia so generally seen in other forms, in which there is a more or less general infection.

All the patients observed were adults or old people, although their calculations showed that the disease may have begun any time in childhood, youth or arlult age. Men and women suffer in equal proportions. Matters of habits or hygiene dicl not appear to be of any consequence. The wearing or not of footgear also did not appear to have any connection with the disease. Occupation had no influence, as field labourers and outside workers suffered the same as inside workers and women. There was nothing to indicate that heredity or cohabitation had any influence. The disease is not endemic in any special area, and the patients came from distinct zones of the country and quote different altitudes and climates.

\section{Clinical picture of paticuts.}

(a) Sul)jective signs were, as already indicated, neuralgic pains, cramps, contractions, heaviness of toes, formication and numbness of toes, diminution and later disappearance of both superficial and (leep sensibility to pain, heat and cold, although sensibility to touch remained.

(b) Objective signs. The patients generally came for examination only after the disease was well advanced. There were noted the changes in the toe nails which were striated, clry, brittle, flattened or curved and deformed: sometimes the nails were found atrophied until only slight traces of them were left adhering to the skin. Such deformities as hammer toe were observed with the parrotfoot-like varus turning in of the feet, changes in the movements of abduction, adduction, extension and flexion, according as the lesions hac affected the cxternal or intcrnal plantar nerves.

The interdigital spaces had disappeared and the toes remained fused together in a solid mass. The mutilation of the toes was without any break in the continuity of the skin, and was due to a process of combined retraction and absorption of the integument and rarefaction of the bones, the distal phalanges becoming cone-shaped stumps, gradually disappearing up to the metatarso-phalangeal joints, at which point the absorption process always definitely stopped. The 
vestiges of the toes remaine( in some as simple buttons of skin, while in others the absorption was so complete that the stumps remained as clean cut as after a surgical operation. The patellar and achilles reflexes remained normal or slightly climinished, but ankle clonus could not be elicited. Siensibility to pain both superficial and (leep) was abolished as well as to heat and cold, although sensibility for touch remained. These signs were found exclusively in the feet and in a few cases up to the lower third of the legs The walk of the patients was peculiar, being slow and mincing, not from any central or spinal lesion, but from the purely mechanical effect of the weight of the feet in the hyperplasia forms; and from the want of support and flexion of the anterior segment of the foot in the cases of multilated toes the patients sometimes looked as if they were walking on stilts or on their heels. Sometimes distinct schlerodermia of the foot was found with drynes's of the skin (anhydrosis). There were also hyperplastic changes in the skin with keloid-like scar formation.

Achromia patches were seen, smooth and anaesthetic, sometimes hyperchromia too, and one case of melanotic pigmentation. There were found pemphigoid lesions which became vesiculo-pustular, ending in ulcers of different aspects, which were sluggish and remained localised; sometimes typical neurotrophic lesions were found, as perforating plantar ulcers, infected and suppurating.

All the lesions had a markedly selective tendency, which is their special characteristic; they were definitely and exclusively confined to the feet, with the skin of the lower thirds of the legs in a condition of elephantiasis. The lesions were always symmetrical in position and simultaneous in their occurrence. No analogous lesions were found in any other part of the body, skin, mucosa or nerves, and no mutilations of the hands or facial paralyses were found. The peripheral circulation was not affected in any way except where the filsrous 'sclerosis of the tissues with the formation of keloid and furrows had impeded the circulation.

Classification of the clinical arieties observed.

(a) The dry variety with no open lesions, but with mutilation of the toes by absorption. (Figs. 4, 6.)

(b) The hyperplastic variety in which the skin and bones of the feet assumed enormous proportions, as in acromegaly, but without the co-existence of deformities in the hands, thorax, craneum, face, etc. In one such case there were some nodlules on the enlarged foot, but no nodules in any other part of the body. (Figs. 1, 2, 3.) 
(c) The mixed variety with (lry mutilation of the toes, sometimes with open sores, atonic discharging ulcers, sometimes even perforating ulcers. (Fig. 5.)

(d) The complex type with clry mutilation of the toes, extensive ulceration of the side of the foot and heel and very pronounced melanotic pigmentation. (Fig. 7.)

Complementary Examinations.

The urine was found normal in all cases; in three cases centrifugal examination revealed the presence of the Hansen bacillus in the sediment fixed by heat and stained with ZiehlNielsen.

Nothing special was noted in the blood.

The serum reactions, Bordet, Wasserman and Kahn, were negative; the Menniche test was positive in three cases. Rubino's reaction of sedimentation was positive.

Nasal mucous examined repeatedly with or without iodine of potassiun, were always negative for Hansen's bacillus.

Smears from open lesions showed the bacillus in two cases.

Material from scrapings of skin of affected parts and from patches of achroma and anaesthetic patches, as well as from gland puncture, gave negative results.

Histopathology.

A nodule from a hyperplasia case was excised and examined anatomo-pathologically. It was found to present all the characteristics of classical lepromes. Similar examinations were made of pieces of tissue from the affected feet. This was considered to be the best method of diagnosis of such clinical cases of neural leprosy, as it did not yield positive restilts in the search for Hansen's bacillus.

Radiograms demonstrated a series of processes taking place in the skeleton of feet, deformation, rarefaction, proliferation and absorption of bone.

In the dry form with mutilation from absorption of the toes, disturbance of sensation and sclerodermia, and administration of chaulmoogra derivatives produced a certain degree of improvement in the return of sensibility to pain, heat and cold, the disappearance of formication, and the feeling of heaviness of the feet, while the skin of the feet became almost quite normal again.

The most marked and most surprising effects, however, were seen in the treatment of mixed cases. In two cases in which the discharge from the local lesion was positive for Hansen's bacillus, the treatment produced complete sterility from bacilli, and complete and firm cicatrisation of the open lesions and even of a perforating ulcer. 

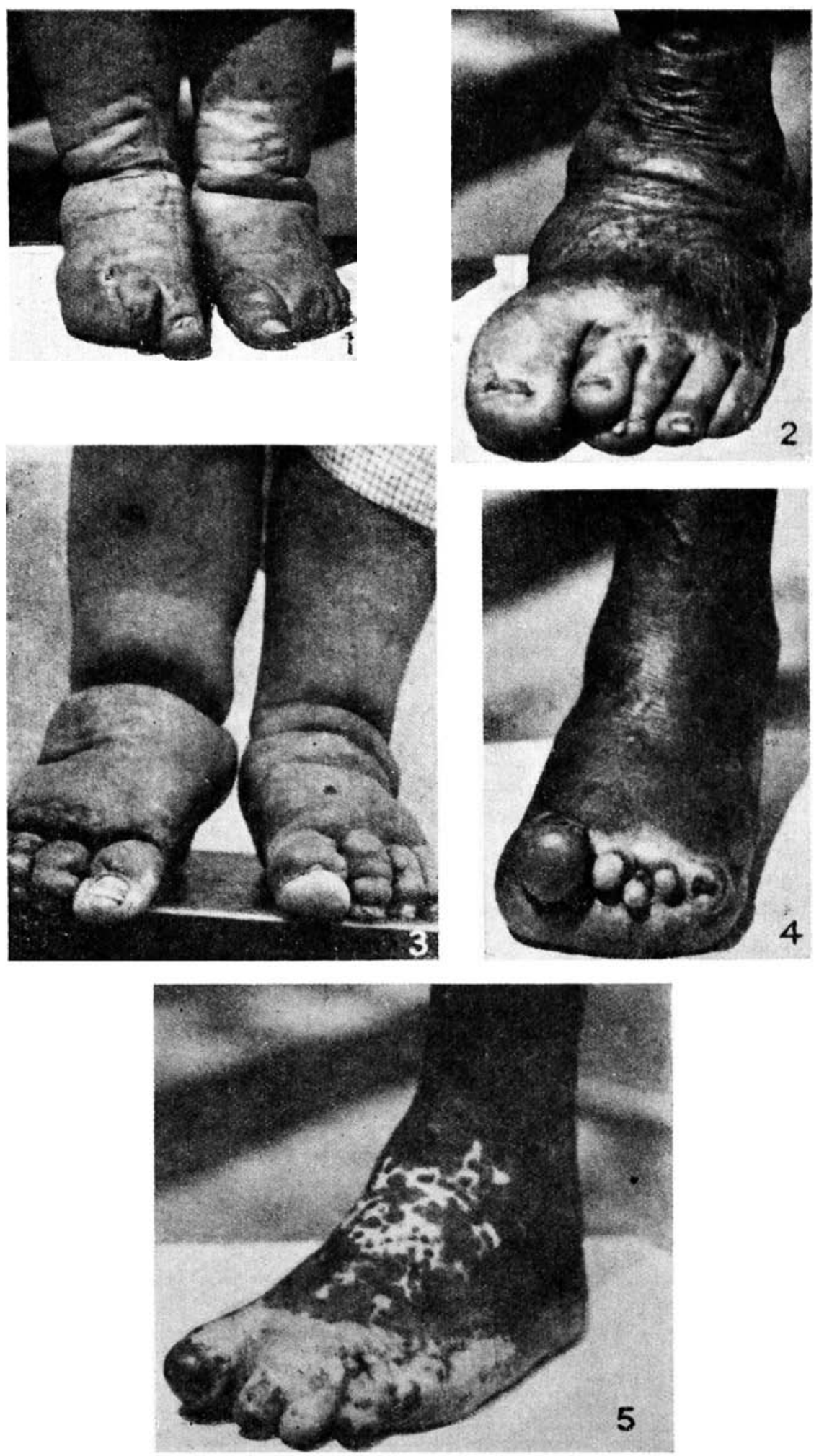

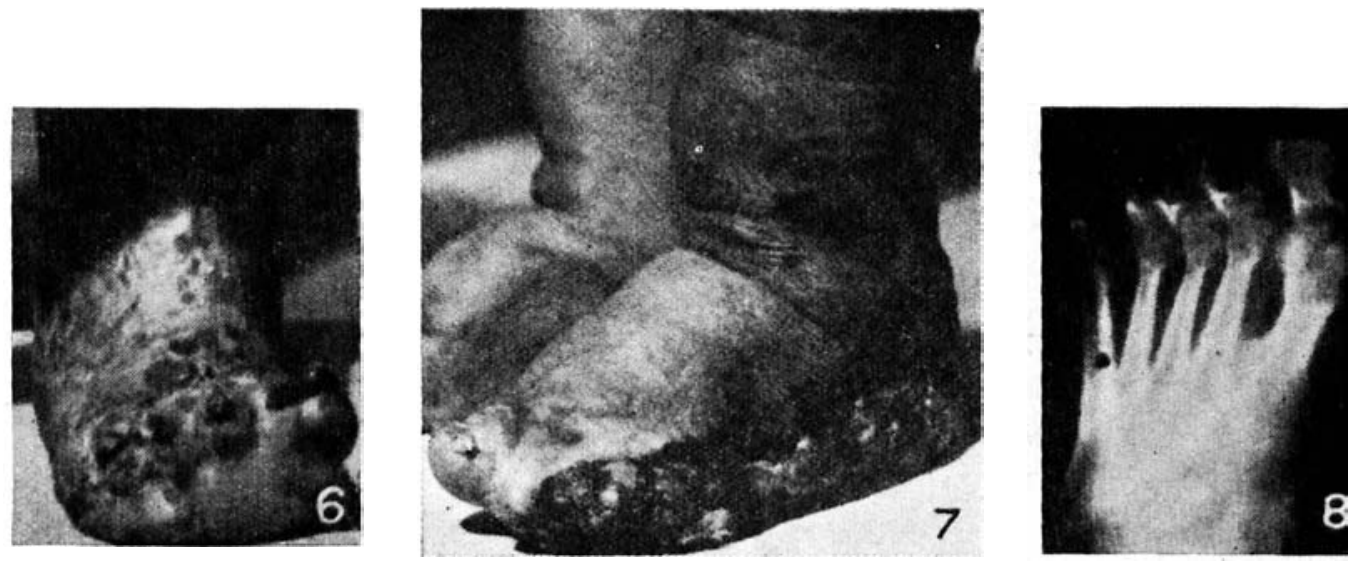

Plates illustrating the cascs.

Fig. 1. Complete mutilation of last four toes of both feet; cutaneous hyperplasia of feet and lower third of legs, very distinct elephantiasis.

Fig. 2. Enormous increase in size of both feet; hyperplasis of skin; change of nails.

Fig. 3. Enormous increase in size of feet; hyperplasia of skin; nodules of skin; achromic anaesthetic patches on big toe of right foot; change in nails; four " hammer toes."

Fig. 4. Mutilation of toes of both feet, which remain as mere buttons of skin. Achromic zones correspond to ulcerated lesions healed after treatment; the patches are anaesthetic.

Fig. 5. Deformity and mutilation of toes; changes in nails; achromic anaesthetic zones corresponding to ulcers cicatrised after treatment; parrot-foot deformity.

Fig. 6. Mutilation of all the toes of both feet; traces of nails left; exfoliative cutaneous lesions; both feet reduced to deformed stumps.

Fig. 7. Mutilation of toes of left foot; remain as simple buttons of skin. Extensive ulceration of outer side of foot as far as the heel, with marked melanotic pigmentation.

Fig. 8. Radiogram showing rarefaction and decalcification of bone. alterations in joints and absorption of phalanges : thickening of metatarsals. 